\title{
Optimal Operation of the Western Link Embedded HVDC Connection
}

\author{
Aaron S. C. Leavy, Waqquas A. Bukhsh, and Keith R. W. Bell \\ Department of Electronic and Electrical Engineering \\ University of Strathclyde \\ Glasgow, United Kingdom \\ \{aaron.leavy, waqquas.bukhsh, keith.bell\}@strath.ac.uk
}

\begin{abstract}
The Western Link is a new point-to-point embedded HVDC connection due to be commissioned in Great Britain in 2018. This paper investigates the optimal loading of the Western Link with respect to the wider transmission system. The work modelled a representation of behaviour of the wholesale market and system operator actions using mathematical optimisation in the form of an economic dispatch followed by an AC optimal power flow. A range of different system cases was studied using: a representative high voltage transmission network of Great Britain; system planned outages on AC circuits in parallel with the Western Link; system contingencies; and two possible post-contingency Western Link loading rules. It was concluded from the cases studied that the optimal dispatch of power on the Western Link is an affine function of power flow in the parallel AC circuits, modulated by system planned outages and the thermal rating of the Western Link.
\end{abstract}

Index Terms-HVDC, OPF, system security and operation

\section{NOMENCLATURE}

Sets
$\mathcal{O}$
$\mathcal{S}$
$\mathcal{G}$
$\mathcal{Z}$
$\mathcal{W}$
$\mathcal{J}$

$\mathcal{H}^{\mathrm{E}}$
$\mathcal{H}^{\mathrm{I}}$
$\mathcal{L}$
$\mathcal{K}$

\section{Parameters}

$P^{\uparrow}, P^{\downarrow}$

Maximum loss of generation, and maximum loss of demand.

$P_{j}^{\uparrow}, P_{j}^{\downarrow}$

Max. ramp up and ramp down in 20 minutes of generator $j$.

$\Gamma_{s}^{\mathrm{W}}, \Gamma_{s}^{\mathrm{D}} \quad$ Total system production of wind, and total demand, under scenario $s$.

$C_{g}^{-}, C_{g}^{+} \quad$ Bid/offer price of generator $g$.

$C_{j} \quad$ Linear cost of generator $j$.

$P_{h} \quad$ Real power infeed of interconnector $h$.

$K_{i} \quad$ Loss coefficient of embedded HVDC link $i$.

\begin{abstract}
$P_{g}^{0} \quad$ Initial set point for generator $g$ in OPF programs $\mathbf{A}, \mathbf{S}$ and $\mathbf{S}^{\prime}$.

$P_{g}^{\mathrm{LB}}, P_{g}^{\mathrm{UB}}$ Min., max. real power outputs of generator $g$.
\end{abstract}

Variables

$p_{j}^{0} \quad$ Real power output of generator $j$ in economic dispatch model E.

$p_{g} \quad$ Real power output of generator $g$.

$\gamma \quad$ Random variable. Representing wind if $\gamma^{\mathrm{W}}$ or demand if $\gamma^{\mathrm{D}}$.

$p_{l, b b^{\prime}}, p_{l, b^{\prime} b}$ Real power flow from busbar $b$ flowing towards busbar $b^{\prime}$ and vice versa into AC transmission circuit $l$.

$p_{i, b b^{\prime}}, p_{i, b^{\prime} b}$ Real power flow into embedded HVDC link $i$, similar to $p_{l, b b^{\prime}}$ and $p_{l, b^{\prime} b}$.

$p_{l}^{\mathrm{AV}} \quad=0.5\left(p_{l, b b^{\prime}}-p_{l, b^{\prime} b}\right)$. Average power flow through AC transmission circuit $l$ from busbar $b$ to busbar $b^{\prime}$.

$p_{i}^{\mathrm{AV}} \quad$ Average power flow through embedded HVDC link $i$, similar to $p_{l}^{\mathrm{AV}}$.

\section{INTRODUCTION}

There is a significant expected future wind generation capacity within Great Britain (GB), much of which has been contracted in recent years. Most of this capacity exists within Scotland and is therefore remote from the majority of significant GB demand centres within England. The need to upgrade the existing transfer capability between Scotland and the rest of GB to ensure effective utilisation of this wind generation capacity has been identified [1]. The GB transmission system owners and operator have identified the Western Link as being a major part of increasing this transfer capability.

The Western Link [2] is a conventional line commutated converter (LCC) HVDC link with a continuous thermal rating of $2250 \mathrm{MW}$. The link is embedded within the GB transmission system, i.e. both converter stations are connected to the same synchronised AC system, as shown in Fig. 1. In addition, the transfer capabilities of the Western Link and the AC circuits operating in parallel with the Link are of the same order of magnitude. This is the first embedded HVDC link in the GB transmission system since the Kingsnorth HVDC Link [3], which was decommissioned in 1987 [4, Sec. 3.1.7]. Given the relative operational inexperience of networks with 


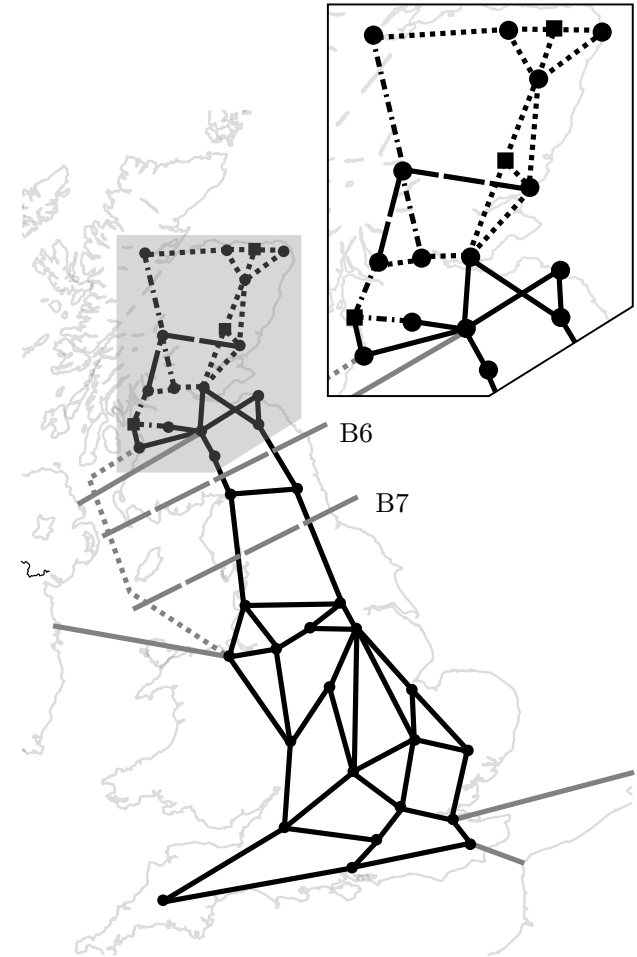

Figure 1. A simplified high voltage GB transmission network indicative route map. Black lines are AC transmission routes: solid are $400 \mathrm{kV}$; dot-dash lines are mixed 275/400 kV; dotted lines are $275 \mathrm{kV}$; and dashed are $132 \mathrm{kV}$ Grey lines: solid are HVDC interconnectors to external markets; dotted is the Western Link; and dashed are transmission boundaries B6 and B7. Black squares: route junctions. Black dots: substations.

embedded HVDC, special consideration must therefore be given to the determination of a steady state power transfer strategy to share power between the Western Link and the parallel AC circuits.

The use of HVDC links to transfer power over large distances with favourable loss characteristics compared to AC transmission has been an accepted solution for many decades. Embedded HVDC links have been historically rare, particularly where links have been expected to operate in partnership with parallel AC circuits of similar power transfer capability. However embedded links are now becoming more common. In Europe, the most recently commissioned embedded link is INELFE [5] which connects the transmission systems of France and Spain, while embedded links within Germany have been proposed [6]-[8].

The spread of offshore transmission networks whether as interconnections, alternative routes within an AC network e.g. the Western Link, or connection of offshore wind farms suggests that HVDC schemes will become more common due to the superiority of HVDC over HVAC in particular offshore applications [9]-[12].

The determination of the dispatch of power on a point-topoint embedded HVDC link has been relatively under-explored in the literature to date. In [13] and [14], particular emphasis is placed on studying a loading strategy for embedded HVDC links based upon an affine function of the voltage phase angles at the embedded links' converter stations' AC busbars.

Minimisation of transmission losses is the objective in [5], [13] and [14], while [13] and [14] also investigate minimisation of power flow through AC circuits. Quadratic approximations are used in [5] to determine a family of curves which may then be used to determine optimal HVDC link transfer set points to minimise transmission losses; this is further discussed in [4, Sec. 4.1.1.2].

An alternative solution method and objective function are studied in [15]: a linearised 'DC'-type OPF is performed with an objective function to maximise the smallest current margin within AC circuits relative to their current ratings; the problem is described as a constrained mixed-integer linear program (MILP) solved using a commercial solver. Only [13] and [15] consider system security explicitly; in particular, [15] models both preventative and corrective security actions. Only [13] and [14] use a selection of system generation and demand scenarios and thus provide significantly different system initial conditions; [5] and [15] do not generally consider different system generation and demand backgrounds.

In general, the methods in [5], [13]-[15] are quite specific: they make assumptions on what the 'best' objectives to minimise are, and typically impose a candidate loading strategy via constraints or objective functions. Satisfying these imposed strategies may lead to optimal solutions which are sub-optimal from the perspective of minimising system generation redispatch cost. They also tend to study a small subset of system generation and demand backgrounds.

We propose a framework which:

1) approximates the market behaviour within a liberalised electricity system to generate credible system states (initial conditions for a transmission system operator) for a range of system operational scenarios and system planned outages;

2) performs full AC optimal power flows on those marketproposed initial conditions to minimise generation redispatch while considering system contingencies and two possible post-contingency operational rules on embedded HVDC links; and

3) infers a loading strategy by inspecting embedded HVDC link and parallel AC circuit loadings across the range of states.

This approach allows consideration of many power system variables across many system scenarios and an understanding of the key factors on which an optimal loading of the embedded HVDC link depends. In addition, the approach also closely approximates the behaviour of liberalised 'net pool' power systems where system operators are tasked with facilitating a market and hence only redispatch system generation and demand from their market-determined positions to ensure satisfactory system operation, security, and quality of supply.

The two operational rules for embedded HVDC link postcontingency loading represent limiting cases of the flexibility in redispatching embedded link transfer following a power system contingency. One rule models minimum flexibility 
where no redispatch of the embedded HVDC link's transfer is possible, and the other rule models maximum flexibility allowing redispatch to anywhere within the link's postcontingency thermal limits.

This paper is organised as follows. In Section II we present our modelling and solution framework. We explain how a standard model of optimal power flow with security constraints can be extended to account for embedded HVDC links. Application of the proposed mathematical programs on a representative GB transmission network is discussed in Section III. Results and discussion are provided in Section IV.

\section{Modelling AND SOlution FRAMEWORK}

The problem we have outlined may be described without reformulation as a non-convex mixed-integer non-linear program (MINLP). The solution of MINLPs is not trivial; these problems may be so computationally challenging that they may be rendered intractable within a reasonable solution time. We propose a framework to decompose the general MINLP into a sequential chain of a MILP and a non-linear program (NLP) in order to lessen the computational burden.

In typical liberalised net pool power systems, market trading occurs between generator and demand parties such that energy demand is satisfied. A system operator is employed to redispatch generators or demands from their market-determined positions and control other power system devices such that the power system may perform within equipment limits and with acceptable levels of security and quality of supply.

Our formulation approximates this process by solving four separate mathematical programs. An economic dispatch problem referred to as $\mathbf{E}$ approximates market trading to determine market positions of schedulable generators to satisfy a specified level of system wind generation and system demand. Optimal power flows (OPFs) referred to as $\mathbf{A}, \mathbf{S}$, and $\mathbf{S}^{\prime}$, represent system operator actions to redispatch market positions of all generation to consider network limits, losses, and network security.

Program A performs an OPF minimising cost of generation redispatch subject to pre-contingency network limits. Program $\mathbf{S}$ models a security constrained OPF with post-contingency flow of the embedded HVDC links constrained by the postcontingency thermal ratings. Program $\mathbf{S}^{\prime}$ performs a security constrained OPF with no change to embedded HVDC link transfer following a contingency except in the contingency of the embedded HVDC link itself, where post-contingency transfer is zero. In Appendix A, the mathematical details of the optimisation programs are provided.

\section{A. Treatment of Stochastic Variables}

Many power systems variables are inherently stochastic. However we constrained the set of stochastic power system variables to contain only two variables: total generation from wind $\gamma^{\mathrm{W}}$; and total system demand $\gamma^{\mathrm{D}}$.

We modelled these stochastic variables by implementing them as parameters $\Gamma_{s}^{\mathrm{W}}$ and $\Gamma_{s}^{\mathrm{D}}$ within our mathematical programs for a particular scenario $s$, and then repeatedly solved these models over $\mathcal{S}:=\left\{s_{i}=\left(\Gamma_{i}^{\mathrm{W}}, \Gamma_{i}^{\mathrm{D}}\right): 1 \leq i \leq n^{\mathrm{S}}\right\}$, where $n^{\mathrm{S}}$ is the total number of scenarios considered.

Note that each scenario was a particular realisation of total production from wind generation and total demand. In this paper, we take a fixed spatial distribution of demand, while the wind farm production was considered to be varying uniformly dependent on a scaling factor and nameplate capacity of each wind farm such that the sum of all wind farms' productions equalled the specified system total wind production. This approach modelled system-wide weather and ignored any localised weather effects.

\section{B. Treatment of Planned Outages}

Assets within power systems such as transformers or transmission circuits require periodic maintenance. Maintenance is usually performed on the asset when it is de-energised; this process usually occurs as part of a planned outage. This means that a power system is unlikely to be operating as an intact system at all times, i.e. planned outages are common. Practical power systems normally contain sufficient flexibility to operate to acceptable system security and quality of supply while multiple planned outages are in place.

We implemented planned outages by repeating each scenario in the OPFs over a set $\mathcal{O}$ of pre-contingency states; note that $\mathcal{O}$ also included a state modelling an intact system. We considered each planned outage state $o \in \mathcal{O}$ by fixing power flow to zero within the equipment under planned outage. Moreover, in this work we only considered planned outages of the transmission assets and not of the generation assets. Note that in our particular formulation, the solution of $\mathbf{E}$ was independent of planned outages since $\mathbf{E}$ assumed a 'copperplate' representation of the power system and thus contained no representation of the network plant we subjected to planned outages. However, a formulation containing planned outages of generator or demand groups would necessarily require explicit modelling of planned outages within $\mathbf{E}$.

\section{Treatment of Economic Dispatch}

The economic dispatch $\mathbf{E}$ committed generators from $\mathcal{J}$ to meet the residual demand $\left(\Gamma_{s}^{\mathrm{D}}-\Gamma_{s}^{\mathrm{W}}\right)$ for $s \in \mathcal{S}$ at minimum total cost of production, subject to $P^{\uparrow}$ and $P^{\downarrow}$ constraints. We de-rated the maximum production capability $P_{g}^{\mathrm{UB}}$ of all schedulable generators by $F$; this small headroom was to ensure schedulable generators could slightly increase their output $p_{g}$ in the OPFs from their economic dispatch position $p_{g}^{0}$ (fixed as $P_{g}^{0}$ within the OPFs) to address system losses and therefore encourage OPF feasibility. We classified schedulable generation as coal, gas, nuclear and hydro units; interconnectors were modelled as fixed injections $P_{h}$ whilst pumped storage units were ignored. ${ }^{1}$

\footnotetext{
${ }^{1}$ The operation of pumped storage is flexible and is largely determined by: its owners' approach to energy arbitrage, and contracts for frequency restoration reserve. Its total capacity is relatively small: approximately $2.7 \mathrm{GW}$ out of a total installed generation capacity of just under $69 \mathrm{GW}$ in our model.
} 


\section{Treatment of Optimal Power Flows}

The model in $\mathbf{E}$ did not consider network constraints or losses, and hence we subsequently performed OPFs to consider these influences. We modelled losses and network constraints within $\mathbf{A}$, while in both programs $\mathbf{S}$ and $\mathbf{S}^{\prime}$ we modelled losses, network and security constraints. The objective of the OPFs sought to minimise the cost of redispatch of all generators within the system with appropriate bid $C_{g}^{-}$and offer $C_{g}^{+}$costs depending on fuel type.

We considered two security constrained OPF formulations to investigate alternative post-contingency transfer rules for embedded HVDC links. Program S allowed HVDC link postcontingency transfer to vary between post-contingency thermal limits, while $\mathbf{S}^{\prime}$ constrained a link's post-contingency power flow to equal its pre-contingency power flow. These two operational regimes represented two extrema of possible operation of an embedded link, and thus allowed us to consider the effect of a link's post-contingency transfer redispatch flexibility on pre-contingency power transfer sharing between the embedded link and parallel AC circuits.

\section{E. Treatment of Equipment Contingencies}

We incorporated equipment contingencies into the security constrained OPF formulations via a direct approach [16], by simultaneously modelling each post-contingency state for each contingency in the list of possible contingencies in conjunction with the pre-contingency state as part of a single mathematical program. We applied a direct approach in contrast with decomposed approaches such as Bender's decomposition [17], [18], as the development time of the direct approach was favourable as well as avoiding potential infeasibility issues due to aggressive cut constraints.

\section{F. Treatment of Embedded HVDC Links}

We approximated the steady state modelling of embedded HVDC links using a 'two-coupled sources' approach. The link's converter stations were modelled as real power sinks $p_{i, b b^{\prime}}$ and $p_{i, b^{\prime} b}$ at the AC busbars $b$ and $b^{\prime}$ at either end of link $i$. The real power flows at each link converter station were linked together via an equality constraint, which forced real power loss within the link to be a quadratic function of the average of the converter real power injections (see (11a) in Appendix A for more details). The real power flow into these sinks was constrained to within bounds, representing the maximum power transfer capability of the link (see (11b) in Appendix A). Note that this approach ignored the disjunctive behaviour of LCC HVDC links' power transfer capability caused by minimum limits on DC-side current.

We did not explicitly model reactive power influences from converter stations or their associated equipment such as shunt compensation or filters. We assumed that power flow into or out of converter stations occurred at unity power factor; in the case of LCC HVDC links, this implies that converter and harmonic filter reactive power consumption and production were perfectly balanced by variable shunt compensation and hence there was no reactive power transfer to or from the $\mathrm{AC}$ network.

\section{G. Program Workflow}

On successful completion of $\mathbf{E}$ for each $s \in \mathcal{S}$, we used the optimal solution from $\mathbf{E}$ on $s$ to initialise schedulable generator commitment and power outputs in $\mathbf{A}, \mathbf{S}$ and $\mathbf{S}^{\prime}$ then solved each OPF for each $(o, s) \in \mathcal{O} \times \mathcal{S}$. Using the results of $\mathbf{A}, \mathbf{S}$ and $\mathbf{S}^{\prime}$ we identified outliers and performed linear regressions on the non-outlying results, where outliers were regarded as scenarios which had parallel power flows which significantly deviated from the linear trend. Algorithm 1 details this regression process, where a single embedded HVDC link $i$ is in parallel operation with multiple parallel AC circuits each indexed by $k$. Outliers were determined by manual inspection of scatter plots of $\left(p_{i}^{\mathrm{AV}}, \sum p_{k}^{\mathrm{AV}}\right)$ pairs.

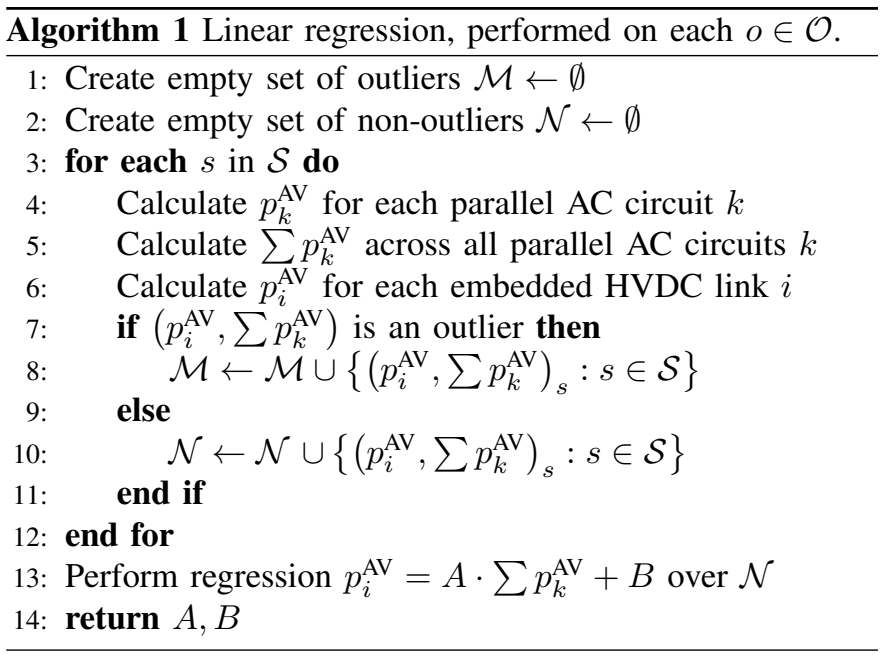

\section{Application to Simplified GB Model}

\section{A. Network Model}

We applied data from a steady state model of the GB transmission network to our framework. Our network model was modified from a version of a GB transmission network transient stability model given as Version 1 in [19]; this transient stability model itself was modified from an earlier steady state GB transmission network model used in [20] and [21] which was developed using principles outlined in [22].

Our model network data included 63 substations: 49, 13, and 1 at $400 \mathrm{kV}, 275 \mathrm{kV}$, and $132 \mathrm{kV}$ voltage levels respectively. There were 62 equivalent generators: 26 represented groups of wind farms, and the remainder represented groups of non-wind generators aggregated by fuel type. The network included $126 \mathrm{AC}$ transmission circuits, and $20 \mathrm{AC}$ transformers.

The model's lumped real power and reactive power losses (which represented 'loading losses' within the equivalenced network), equivalent network shunt admittances, and real and reactive group demands were initialised as Scenario 0 in [19]. When applying different total system demands, we scaled demand groups linearly, scaled lumped real losses 
quadratically, and scaled each lumped reactive loss to maintain a constant power factor equal to the reactive loss' power factor in Scenario 0. Shunt admittances remained unmodified, as these modelled 'no-loading losses' within equivalent networks.

We now note the specific changes from [19]. The $400 \mathrm{kV}$ circuit configuration in Scotland was changed to reflect the removal of Inverkip substation. The pre-contingency and postcontingency ratings of AC transmission circuits were calculated by taking $84 \%$ and $106.5 \%$ of the winter post-contingency ratings in [19]. The installed capacity of generators was updated using data in [23]. Generator transformers were ignored, so generators were connected directly to busbars. ${ }^{2}$ Series capacitors were assumed to be in service and maintained at a constant series reactance at all times. The Western Link was modelled using the 'two-coupled sources' approach: the EastWest and Moyle interconnectors were assumed to be at 'float', i.e. with $0 \mathrm{MW}$ transfer; and BritNed and IFA interconnectors were set to constant maximum import.

The network switched shunt and variable reactive compensation equipment at each busbar were approximated as an ideal continuously variable shunt reactive compensator at each busbar, with upper and lower bounds set equal to the maximum and minimum reactive power injection possible from the combinations of switched and variable compensation. The busbar voltage magnitude upper and lower bounds were set to $\pm 0.15 \mathrm{pu}$ and $\pm 0.3 \mathrm{pu}$ from unity in pre-contingency and post-contingency states respectively. ${ }^{3}$

\section{B. Demand and Generation Background Assumptions}

We defined minimum and maximum possible realisations of demand and wind within the GB network. These bounds were used to create scenarios for our deterministic models. We used $18100 \mathrm{MW}$ and $51511 \mathrm{MW}$ as minimum and maximum possible realisations of total demand for the GB network respectively using historic data from [24] and [25]. For wind power generation, we used $0 \%$ and $100 \%$ of wind generation capacity from [23]. Using these bounds, we generated a total of 196 scenarios, consisting of 14 total system demands and 14 total system wind productions; both sets uniformly sampled system demands and system wind productions between the aforementioned limits inclusively.

We recorded power flow variables for the Western Link, four AC circuits crossing the B6 boundary, and four AC circuits crossing the B7 boundary; the locations of these boundaries are shown in Fig. 1. Each of the single black lines shown in Fig. 1 across B6 and B7 boundaries consist of two AC circuits. The Western Link crosses both B6 and B7 boundaries, hence we performed the regression analysis on both boundaries.

We considered 5 planned outages plus the intact system. The planned outages consisted of various $N-1$ and $N-D$ outages of AC circuits over boundary B6 in parallel with the

\footnotetext{
${ }^{2}$ Equivalent generator real and reactive bounds should normally be altered to reflect this change but this was not done in this study in view of the relatively minor influence.

${ }^{3}$ These represented relaxed limits relative to normal operational limits, a choice made in view of the approximate nature of the representation of the GB system and in order to encourage OPF feasibility.
}

Western Link. State 'Int' represented an intact system with no planned outages. States 'EC1' and 'WC1' represented planned outages of one circuit only in the East and West Coast AC B6 double circuit routes respectively. States 'EC2' and 'WC2' were similar to 'EC1' and 'WC1' except they modelled outages of both circuits in a route. State 'EW1' represented planned outages of one circuit in each of the East and West Coast AC B6 double circuit routes simultaneously. Hence the set $\mathcal{O}$ members were $\{$ Int, EC1, WC1, EC2, WC2, EW 1$\}$.

Losses in the Western Link were modelled as a quadratic function of the Link's average power flow. We calculated the quadratic component of loss function $K$ for the Western Link by assuming a loss of $45 \mathrm{MW}$ from [26] at $2250 \mathrm{MW}$ flow into the rectifier end of the link.

In $\mathbf{E}$, we used short run marginal cost data from [27] to model cost of production $C_{j}$ per generation fuel type, and assumed $P_{j}^{\uparrow}$ and $P_{j}^{\downarrow}$ per fuel type from [28]. Total $P^{\uparrow}$ and $P^{\downarrow}$ were modelled as $1.32 \mathrm{GW}$ and $1.5 \mathrm{GW}$ respectively. In $\mathbf{A}, \mathbf{S}$ and $\mathbf{S}^{\prime}$, we used bid and offer cost data for $C_{g}^{-}$and $C_{g}^{+}$from [27].

We described $\mathbf{E}$ using Microsoft Excel and OpenSolver [29], and $\mathbf{A}, \mathbf{S}$, and $\mathbf{S}^{\prime}$ using models written in Pyomo [30] using the Python programming language. We solved Program $\mathbf{E}$ using the CBC solver [31], and Programs $\mathbf{A}, \mathbf{S}$, and $\mathbf{S}^{\prime}$ using the IPOPT solver [32]. We used default settings for both solvers.

\section{Contingencies}

All contingencies were either $N-1$ or $N-D$ which modelled loss of a single item of equipment or a double circuit overhead line. We modelled loss of generators, transformers, and circuits to the north of Melksham, Sundon, East Claydon, Pelham and Walpole. We also modelled the complete loss of the Western Link itself. Where a circuit formed one of a pair of circuits in a double circuit route, we ignored the single circuit contingencies of both individual circuits and modelled only the double circuit contingency of both circuits representing the loss of the double circuit route. Since generators within the model represented a grouping of nearby generators by fuel type, we modelled generator contingencies within a group by reducing the post-contingency real and reactive power upper bounds in the group's equivalent generator to model the loss of the largest single infeed within the generator group.

\section{RESUlTS AND Discussion}

A total of 36 linear regressions (product of 6 precontingency states, 3 OPFs, and 2 boundaries) of HVDC average transfer and total AC boundary average transfer were generated. All 36 regressions indicated identical qualitative behaviour: most pre-contingency HVDC and AC boundary transfers followed an affine trend $p_{i}^{\mathrm{AV}}=A \cdot \sum p_{k}^{\mathrm{AV}}+B$ where coefficient $A$ and offset $B$ were found via the regression, before levelling off at the maximum thermal rating of the Western Link. Fig. 2 illustrates the positions of outliers, nonoutliers, and a fitted affine trend line of boundary transfers for a particular OPF and pre-contingency state; 35 plots matching 
TABLE I

COEFFICIENTS $A$ FROM REgRESSIONS

\begin{tabular}{llllllll}
\hline \multirow{2}{*}{ Boundary } & \multirow{2}{*}{ OPF } & \multirow{6}{*}{ System Pre-Contingency State } \\
& & Int & EC1 & WC1 & EC2 & WC2 & EW1 \\
\hline B6 & A & 0.584 & 0.654 & 0.673 & 1.101 & 0.963 & 0.761 \\
B6 & $\mathbf{S}^{\prime}$ & 0.594 & 0.659 & 0.689 & 1.092 & 1.028 & 0.764 \\
B6 & $\mathbf{S}$ & 0.584 & 0.651 & 0.673 & 1.098 & 0.969 & 0.751 \\
\hline B7 & $\mathbf{A}$ & 0.491 & 0.545 & 0.559 & 0.787 & 0.751 & 0.626 \\
B7 & $\mathbf{S}^{\prime}$ & 0.491 & 0.554 & 0.568 & 0.789 & 0.78 & 0.628 \\
B7 & $\mathbf{S}$ & 0.493 & 0.547 & 0.556 & 0.78 & 0.72 & 0.623 \\
\hline
\end{tabular}

TABLE II

OFFSETS $B$ FROM REGRESSIONS

\begin{tabular}{llllllll}
\hline \multirow{2}{*}{ Boundary } & \multirow{2}{*}{ OPF } & \multirow{5}{*}{ Int } & EC1 & WC1 & EC2 & WC2 & EW1 \\
\hline B6 & A & -28.5 & 4.00 & -47.9 & 220 & -114 & -5.13 \\
B6 & S $^{\prime}$ & -55.4 & -19.9 & -90.7 & 209 & -233 & -28.6 \\
B6 & S & -29.6 & 6.42 & -52.7 & 232 & -132 & 13.1 \\
\hline B7 & A & 358 & 428 & 397 & 902 & 528 & 491 \\
B7 & $\mathbf{S}^{\prime}$ & 339 & 404 & 369 & 883 & 520 & 460 \\
B7 & S & 353 & 434 & 402 & 892 & 581 & 494 \\
\hline
\end{tabular}

the other 35 regressions had qualitatively similar outlier, nonoutlier and trend line behaviours as those shown in Fig. 2.

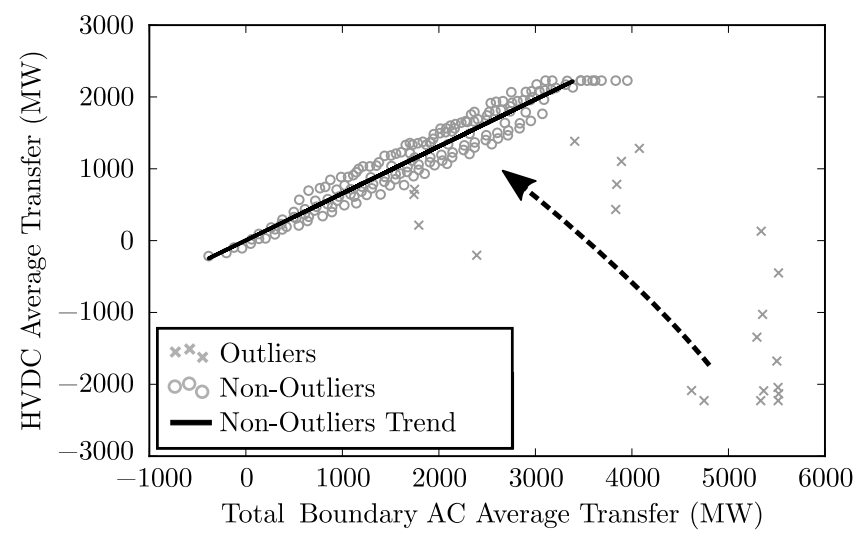

Figure 2. Scatter plot of HVDC and total parallel AC average transfers from the optimal solutions of the feasible scenarios for program A, boundary B6, and pre-contingency system state 'EC1'. The arrow shows the direction of the trend of increasing total system demand level; outliers with higher system demands were much closer to the trend line. Positive transfer indicates flow from Scotland to England and Wales.

Some scenarios (referred to previously as outliers) fell 'below' these group trends: in the outliers, the Western Link transfer was substantially less than other non-outlying scenarios for the same AC transfer. Most of these outliers contained $\Gamma_{s}^{\mathrm{D}}<23 \mathrm{GW}$, and were common across all OPFs and pre-contingency states. This suggests that the outlier behaviour was caused by the generation commitment from the economic dispatch problem. Tables I and II summarise the fitted coefficients and offsets of the affine trends calculated for all 36 regressions.

The coefficients $A$ in Table I are of great significance. For example: the intact system case (indicated by 'Int' in Tables I
TABLE III

PRE-CONTINGENCY DC THERMAL RATING AS A PROPORTION OF TOTAL PARALLEL AC CIRCUITS PRE-CONTINGENCY THERMAL RATING

\begin{tabular}{lllllll}
\hline Boundary & Int & EC1 & WC1 & EC2 & WC2 & EW1 \\
\hline B6 & 0.28 & 0.394 & 0.355 & 0.666 & 0.483 & 0.56 \\
\hline
\end{tabular}

and II) in A over boundary B6 indicates that the HVDC link transfer should be approximately $58 \%$ of the total transfer through the $\mathrm{AC}$ circuits, ignoring offset $B$; hence in this case $1 \mathrm{MW}$ of power from Scotland to England should be shared approximately: $37 \%$ on HVDC and $63 \%$ on the AC, again ignoring offset $B$.

The sharing indicated by coefficients $A$ in Table I can be compared with sharing based only upon boundary thermal capabilities of the HVDC and AC circuits. The thermal capability of the HVDC link as a proportion of the total AC thermal capabilities are given in Table III. If sharing based upon proportion of boundary thermal rating capability, the HVDC link would carry $28 \%$ of the total B6 AC transfer in the 'Int' case i.e. $1 \mathrm{MW}$ of power from Scotland to England should be shared approximately: $22 \%$ on HVDC and $78 \%$ on the AC. The values in Table I are substantially greater than those in Table III for the corresponding pre-contingency state. In other words, the HVDC link is more heavily loaded relative to its rating than the $\mathrm{AC}$ circuits.

The loading on the Western Link increases with decreasing transfer capacity on the AC side. The values of offsets $B$ in Table II depend on the scenario initial conditions.

From Table I, considering system security via OPFs $\mathbf{S}$ and $\mathbf{S}^{\prime}$ did not significantly influence values of coefficient $A$ across the regressions. In addition, the extrema of operation governing Western Link post-contingency transfer did not influence pre-contingency parallel power sharing coefficients either. The only significant influence over trend line coefficients came from considering planned outages of parallel circuits over boundary B6. This suggests that system security did not affect these offsets significantly, but Western Link postcontingency flexibility did have a significant effect. Hence the Western Link should carry a higher proportion of the precontingency boundary transfer if the Link can be redispatched following system contingencies. This additional Link transfer in the pre-contingency state is not dependant on the precontingency transfer in the parallel AC circuits.

Note from Fig. 2 that some outliers have significant bidirectional flows in parallel circuits leading to loop flows through the Western Link and the parallel AC circuits. An initial study of the results has not revealed what effect was causing these loop flows.

One hypothesis is that these loop flows were occurring as a zero cost method to increase system losses to help consume excess real power from generators in the OPFs due to excessive market positions $P_{g}^{0}$ generated from $\mathbf{E}$ solutions. This may have occurred if excess generation was committed where the cost of reducing that generation was positive to the objective function, for example with wind or nuclear generation; hence 
wasting excess generation by increasing system losses was more cost effective than paying to reduce generation output.

This phenomenon would tend to lead to maximum loop flows since the increase in losses is not particularly large in the network for large loop flows, and would occur for even small over-commitments of generation in the order of tens of megawatts. Over commitments in $\mathbf{E}$ may have occurred due to the strenuous loss of infeed and outfeed parameters; for low system demands, the ramping to meet loss of infeed and outfeed has to be met by schedulable generation only due to Program $\mathbf{E}$ formulation where wind generation could not partake in ramping. Since almost all schedulable generators had non-zero production limits in $\mathbf{E}$, this meant that schedulable generators may have been committed to meet the ramping constraints in $\mathbf{E}$ but simultaneously causing excess real power production compared to the system demand level.

\section{CONCLUSION}

The problem of determination of a suitable steady state loading strategy of embedded point-to-point HVDC links has been identified. The projected increase in the relevance of this problem is highlighted. Previous research has been summarised before proposing a solution framework developed using a more generalised approach than preceding work. Our method relies on optimal power flows on a large number of operational scenarios to provide data which we then use to infer a suitable embedded link transfer strategy. We explored two alternative rules on the embedded HVDC link's postcontingency transfer; these rules modelled limiting cases on the flexibility of modulating the post-contingency transfer. We apply our solution framework to a model of the GB transmission system to determine a candidate loading strategy for the Western Link, whilst considering credible system contingencies, pre-contingency states, system demand and wind generation operational scenarios, and two alternative embedded HVDC link post-contingency transfer constraints.

The results of this analysis indicate that Western Link transfers share an affine trend with the total transfer in parallel AC circuits. System security and embedded HVDC link postcontingency transfer flexibility do not significantly influence these affine trend lines. In initially intact network conditions, the pre-contingency dispatch of power on the HVDC link is the same whether post-contingency redispatch of the link's power transfer is possible or not suggesting that short-term post-contingency redispatch is not necessary for the contingencies considered; some action to re-secure the system would generally be required in practice. However pre-contingency states that included planned network outages did affect the affine trend coefficients and offsets. These implied that, when post-contingency redispatch of the HVDC link is possible, it should initially carry more of the total power transfer. This, in turn, suggests that the critical post-contingency action is a reduction in the HVDC link's loading. We found that the only non-uniform behaviour exhibited other than that previously described was related to low system demand conditions, where significant loop flows were found.
These results are not the product of a final resolution to the problem of optimal steady state loading of embedded HVDC links. There is significant further work required before system asset owners and operators can be fully confident in either our proposed framework or our results.

This paper's results leads to two immediate avenues of possible further enquiry: consideration of space-dependant wind generation production, and explanation of outlier scenarios. The former would allow modelling of weather fronts and realistic spatial correlation between wind outputs of less then unity, whilst the latter would indicate if the outliers in our results are indeed a manifestation of surplus negative bid cost generation as we hypothesise.

One shortcoming of our framework is its overly simplified treatment of generation. Our framework may be improved by including non-zero generator real power minimum limits in the OPFs, and constraining the ramping of generators' outputs between pre-contingency and post-contingency states.

We selected sets of planned outages and contingencies based upon engineering judgement. Further work could focus on detecting other relevant planned outages and contingencies which may affect power sharing. Sensitivity analyses based upon distribution factors may provide a useful framework with which to detect sets of important generators, demands and transmission circuits. These methods may even provide explanation as to the magnitudes of the differences of sharing coefficients and offsets we have observed in this paper. However these sensitivity methods are typically based upon linearised 'DC'-type programs, hence extending them to nonconvex NLP and MILP programs would be non-trivial.

Our generation and demand scenarios in conjunction with space-independent wind production resulted in the vast majority of cases giving power transfer from Scotland towards England and Wales. Further work is needed to determine optimal transfer sharing of net flows into Scotland. Such net flows may be encountered during low wind, high demand times in conjunction with unplanned outages of schedulable generation within Scotland.

\section{APPENDIX A}

\section{PROGRAM FORMULATIONS}

\section{A. Economic Dispatch}

Program $\mathbf{E}$ is described by objective function (1) subject to constraints (2a)-(2f), $\forall j \in \mathcal{J}$. Although $\mathbf{E}$ is repeated $\forall s \in \mathcal{S}$, we have dropped index $s$ for brevity. Note that parameters $\Gamma^{\mathrm{W}}$ and $\Gamma^{\mathrm{D}}$ are fixed to particular values $\Gamma^{\mathrm{W}}:=\left(\gamma_{s}^{\mathrm{W}}\right)^{*}$ and $\Gamma^{\mathrm{D}}:=$ $\left(\gamma_{s}^{\mathrm{D}}\right)^{*}$ depending on $s$.

$$
\min \sum_{j} C_{j} p_{j}^{0}
$$

subject to

$$
\begin{gathered}
\sum_{j} p_{j}^{0} \geq \Gamma^{\mathrm{D}}-\Gamma^{\mathrm{W}} \\
\sum_{j} p_{j}^{\downarrow} \geq P^{\downarrow}, \quad \sum_{j} p_{j}^{\uparrow} \geq P^{\uparrow}
\end{gathered}
$$




$$
\begin{gathered}
\mu_{j}, \mu_{j}^{\uparrow}, \mu_{j}^{\downarrow} \in\{0,1\}, \quad \mu_{j}^{\uparrow}, \mu_{j}^{\downarrow} \leq \mu_{j} \\
0 \leq p_{j}^{\uparrow} \leq \mu_{j}^{\uparrow} P_{j}^{\uparrow}, \quad 0 \leq p_{j}^{\downarrow} \leq \mu_{j}^{\downarrow} P_{j}^{\downarrow} \\
\mu_{j} P_{j}^{\mathrm{LB}} \leq p_{j}^{0}+p_{j}^{\uparrow} \leq \mu_{j} F P_{j}^{\mathrm{UB}} \\
\mu_{j} P_{j}^{\mathrm{LB}} \leq p_{j}^{0}-p_{j}^{\downarrow} \leq \mu_{j} F P_{j}^{\mathrm{UB}}
\end{gathered}
$$

\section{B. Non-Security Constrained OPF}

Program $\mathbf{A}$ is described by objective function (3) subject to constraints (4a)-(15). Note that parameters $M_{g}$ and $P_{g}^{0}$ were set from the corresponding optimal values of the variables $\mu_{g}$ and $p_{g}^{0}$ for generator $g \in \mathcal{J}$ in $\mathbf{E}$ by $M_{g}:=\left(\mu_{g}\right)^{*}$ and $P_{g}^{0}:=$ $\left(p_{g}^{0}\right)^{*}$. For generator $g \in \mathcal{W}, M_{g}$ and $P_{g}^{0}$ were set as functions of $\Gamma^{\mathrm{W}}$ as discussed in Section II-A. In Programs $\mathbf{A}, \mathbf{S}$, and $\mathbf{S}^{\prime}$, demands and lumped losses were modelled as a unified set of loads with parameters real $P_{d}$ and reactive $Q_{d}$ powers set as a function of $\Gamma^{\mathrm{D}}$ as discussed in Section II-A.

$$
\min \sum_{g \in \mathcal{G}}\left(C_{g}^{+} \Delta p_{g}^{+}-C_{g}^{-} \Delta p_{g}^{-}\right)
$$

The increments and decrements $\Delta p_{g}^{+}$and $\Delta p_{g}^{-}$from the market position $P_{g}^{0}$ of generator $g$ were linked together with the generator's $p_{g}$ and $P_{g}^{0}$ by (4a), $\forall g \in \mathcal{G}$; (4b) ensured that generator $g$ increments and decrements were nonnegative $\forall g \in \mathcal{G}$.

$$
\begin{gathered}
p_{g}=P_{g}^{0}+\left(\Delta p_{g}^{+}-\Delta p_{g}^{-}\right) \\
\Delta p_{g}^{+}, \Delta p_{g}^{-} \geq 0
\end{gathered}
$$

The real and reactive power balances at each busbar $b$ were constrained by (5a) and (5b), $\forall b \in \mathcal{B}$ where $\mathcal{B}$ is the set of all network busbars.

$$
\begin{aligned}
& \sum_{g \in \mathcal{G}_{b}} p_{g}+\sum_{h \in \mathcal{H}_{b}^{E}} P_{h}= \\
& \sum_{d \in \mathcal{D}_{b}} P_{d}+\sum_{\substack{l \in \mathcal{L}_{b} \\
b^{\prime} \in \mathcal{B}_{l} \\
b \neq b^{\prime}}} p_{l, b b^{\prime}}+\sum_{\substack{i \in \mathcal{H}_{b}^{I} \\
b^{\prime} \in \mathcal{B}_{i} \\
b \neq b^{\prime}}} p_{i, b b^{\prime}}+\sum_{\substack{t \in \mathcal{T}_{b} \\
b^{\prime} \in \mathcal{B}_{t} \\
b \neq b^{\prime}}} p_{t, b b^{\prime}}+G_{b} v_{b}^{2} \\
& \sum_{g \in \mathcal{G}_{b}} q_{g}+\sum_{z \in \mathcal{Z}_{b}} q_{z}= \\
& \sum_{d \in \mathcal{D}_{b}} Q_{d}+\sum_{\substack{l \in \mathcal{L}_{b} \\
b^{\prime} \in \mathcal{B}_{l} \\
b \neq b^{\prime}}} q_{l, b b^{\prime}}+\sum_{\substack{t \in \mathcal{T}_{b} \\
b^{\prime} \in \mathcal{B}_{t} \\
b \neq b^{\prime}}} q_{t, b b^{\prime}}-B_{b} v_{b}^{2}
\end{aligned}
$$

The real and reactive power flow from busbar $b$ into each transmission circuit $l$ linking busbars $b$ and $b^{\prime}$ where $\left\{b, b^{\prime}\right\}=$ $\mathcal{B}_{l}$ are given by (6a) and $(6 \mathrm{~b}), \forall l \in \mathcal{L}$. Similar flows from busbar $b^{\prime}$ into $l$ also applied; these may be found by negating $\left(\theta_{b}-\theta_{b^{\prime}}\right)$ in (6a) and (6b). Similar real and reactive flow from busbars $b$ and $b^{\prime}$ into each transformer $t \in \mathcal{T}$ where $\left\{b, b^{\prime}\right\}=\mathcal{B}_{t}$ applied but with variable $g_{t, b b}, b_{t, b b}, g_{t, b^{\prime} b^{\prime}}$, $b_{t, b^{\prime} b^{\prime}}, g_{t, b b^{\prime}}, g_{t, b^{\prime} b}, b_{t, b b^{\prime}}$, and $b_{t, b^{\prime} b}$ where applicable.

$$
\begin{aligned}
& p_{l, b b^{\prime}}=v_{b}^{2} G_{l}- \\
& \quad v_{b} v_{b^{\prime}}\left[G_{l} \cos \left(\theta_{b}-\theta_{b^{\prime}}\right)+B_{l} \sin \left(\theta_{b}-\theta_{b^{\prime}}\right)\right]
\end{aligned}
$$

$$
\begin{aligned}
q_{l, b b^{\prime}}=- & v_{b}^{2} B_{l}^{\prime}+ \\
& v_{b} v_{b^{\prime}}\left[B_{l} \cos \left(\theta_{b}-\theta_{b^{\prime}}\right)-G_{l} \sin \left(\theta_{b}-\theta_{b^{\prime}}\right)\right]
\end{aligned}
$$

Constraint (7) ensured that the thermal rating of each transmission circuit $l$ was not exceeded at the busbar $b$ end of the circuit, with a similar constraint at the busbar $b^{\prime}$ end. Similar constraints existed for each transformer $t \in \mathcal{T}$.

$$
p_{l, b b^{\prime}}^{2}+q_{l, b b^{\prime}}^{2} \leq S_{l}^{2}
$$

Variable $g_{t, b b}$ and $b_{t, b b}$ of transformer $t$ at busbar $b$ are given by (8). Similar constraints on $g_{t, b^{\prime} b^{\prime}}$ and $b_{t, b^{\prime} b^{\prime}}$ at busbar $b^{\prime}$ applied, and may be found by substituting $\tau_{t}^{2}$ with 1 in (8).

$$
\left(g_{t, b b}, b_{t, b b}\right)=\left(\frac{G_{t}}{\tau_{t}^{2}}, \frac{B_{t}}{\tau_{t}^{2}}\right)
$$

Variable $g_{t, b b^{\prime}}$ and $b_{t, b b^{\prime}}$ of transformer $t$ viewed from busbar $b$ are given by (9a) and (9b). Similar constraints on $g_{t, b^{\prime} b}$ and $b_{t, b^{\prime} b}$ at busbar $b^{\prime}$ applied, and may be found by negating $\alpha_{t}$ in (9a) and (9b).

$$
\begin{aligned}
g_{t, b b^{\prime}} & =-\frac{1}{\tau_{t}}\left[G_{t} \cos \left(\alpha_{t}\right)-B_{t} \sin \left(\alpha_{t}\right)\right] \\
b_{t, b b^{\prime}} & =-\frac{1}{\tau_{t}}\left[B_{t} \cos \left(\alpha_{t}\right)+G_{t} \sin \left(\alpha_{t}\right)\right]
\end{aligned}
$$

Tap ratios $\tau_{t}$ and phase shifts $\alpha_{t}$ of transformer $t$ were bounded by upper and lower bounds by (10).

$$
\left(T_{t}^{\mathrm{LB}}, A_{t}^{\mathrm{LB}}\right) \leq\left(\tau_{t}, \alpha_{t}\right) \leq\left(T_{t}^{\mathrm{UB}}, A_{t}^{\mathrm{UB}}\right)
$$

Real power losses in embedded HVDC link $i$ were constrained to vary quadratically with the average real power transfer in the link as given by (11a), $\forall i \in \mathcal{H}^{I}$. Constraint (11b) ensured that real power flow from busbar $b$ into embedded HVDC link $i$ did not exceed the link's thermal rating, with a similar constraint applying at busbar $b^{\prime}$.

$$
\begin{gathered}
p_{i, b b^{\prime}}+p_{i, b^{\prime} b}=K_{i}\left(\frac{p_{i, b b^{\prime}}-p_{i, b^{\prime} b}}{2}\right)^{2} \\
-S_{i} \leq p_{i, b b^{\prime}} \leq S_{i}
\end{gathered}
$$

Constraints (12a)-(12b) ensured that the real and reactive power production of generator $g$ were within upper and lower bounds. Note that $P_{g}^{\mathrm{UB}}$ was set as a function of $\Gamma^{\mathrm{W}}$ for all wind generators $g \in \mathcal{W}$.

$$
\begin{aligned}
& 0 \leq p_{g} \leq M_{g} P_{g}^{\mathrm{UB}} \\
& Q_{g}^{\mathrm{LB}} \leq q_{g} \leq Q_{g}^{\mathrm{UB}}
\end{aligned}
$$

Similar to generators, (13) ensured that the reactive power production of shunt compensator $z$ was within acceptable upper and lower bounds, $\forall z \in \mathcal{Z}$.

$$
Q_{z}^{\mathrm{LB}} \leq q_{z} \leq Q_{z}^{\mathrm{UB}}
$$

To avoid redundancy, the voltage phase angle of a selected reference bus $b$ where $b \in \mathcal{B}_{0}$ is given by (14).

$$
\theta_{b}=0
$$

Busbar $b$ voltage magnitude was constrained by upper and lower bounds by (15), $\forall b \in \mathcal{B}$.

$$
V_{b}^{\mathrm{LB}} \leq v_{b} \leq V_{b}^{\mathrm{UB}}
$$




\section{Security Constrained OPFs}

Programs $\mathbf{S}$ and $\mathbf{S}^{\prime}$ included the same objective function (3) and constraints (4a)-(15) as $\mathbf{A}$; in $\mathbf{S}$ and $\mathbf{S}^{\prime}$, (4a)-(15) modelled constraints in the pre-contingency state. Programs $\mathbf{S}$ and $\mathbf{S}^{\prime}$ also consisted of extra variables and constraints to model each post-contingency state $c$ within the set of contingencies $\mathcal{C}$. The variables and constraints in each post-contingency state $c$ were similar to the pre-contingency variables and constraints in (4a)-(15), except:

- where the state $c$ modelled the contingency of a piece of equipment $e$;

- treatment of post-contingency real power flow in embedded HVDC links; and

- treatment of post-contingency tap ratio and phase shift of transformers

If a state $c$ modelled the loss of equipment $e$ excluding generators, the relevant real and reactive flow variables of equipment $e$ were constrained to zero thus modelling the loss of equipment $e$. Contingencies of generators were modelled via reducing the generators' real and reactive power upper bounds. Transformer post-contingency tap ratios and phase shifts were constrained such that they equalled precontingency tap ratios and phase shift angles respectively. For embedded HVDC links specifically, (16a) and (16b) applied to Programs $\mathbf{S}$ and $\mathbf{S}^{\prime}$ respectively in post-contingency states which did not model the loss of the embedded link itself. The link flow was constrained to zero in both Programs in those post-contingency states which did model the contingency of the embedded link.

$$
\begin{gathered}
-S_{c, i} \leq p_{c, i, b b^{\prime}} \leq S_{c, i} \\
p_{c, i, b b^{\prime}}=p_{i, b b^{\prime}}
\end{gathered}
$$

\section{REFERENCES}

[1] "Our electricity transmission network: A vision for 2020 full report," Electricity Networks Strategy Group, Tech. Rep., 2009.

[2] S. Achenbach, V. Barry, C. H. Bayfield, and P. F. Coventry, "Increasing the GB electricity transmission networks' power transfer capability between North and South - The Western HVDC Link," in 10th IET International Conference on AC and DC Power Transmission, Dec. 2012, pp. 1-4

[3] T. E. Calverley, A. Gavrilovic, F. H. Last, and C. W. Mott, "The Kingsnorth-Beddington-Willesden D.C. Link," in CIGRE International Conference on Large High Tension Electric Systems, no. 43-04, Jun. 1968, pp. 1-14.

[4] JWG C4/B4/C1-604, "Influence of embedded HVDC transmission on system security and AC network performance," CIGRE, Paris, Tech. Brochure 536, 2013.

[5] P. L. Francos, S. S. Verdugo, H. F. Álvarez, S. Guyomarch, and J. Loncle, "INELFE - Europe's first integrated onshore HVDC interconnection," in IEEE Power and Energy Society General Meeting, Jul. 2012, pp. 1-8.

[6] W. H. Wellssow and H. Pluntke, "Planning the optimal number and placement of embedded VSC-HVDC links in the German AC transmission grid," in International ETG Congress, Nov. 2015, pp. 1-7.

[7] E. Spahic, F. Schettler, D. Varma, and J. Dorn, "Impact of the DC technology on transmission grids," in 11th IET International Conference on AC and DC Power Transmission, Feb. 2015, pp. 1-7.

[8] J. Dorn, M. Pohl, D. Retzmann, and F. Schettler, "Transformation of the energy system in Germany - Enhancement of system stability by integration of innovative multilevel HVDC in the AC grid," in International ETG Congress, Nov. 2013, pp. 1-6.
[9] D. Elliott et al., "A comparison of AC and HVDC options for the connection of offshore wind generation in Great Britain," IEEE Transactions on Power Delivery, vol. 31, no. 2, pp. 798-809, April 2016.

[10] S. Kolluri, P. Thummala, R. Sapkota, S. K. Panda, and D. Rendusara, "Subsea power transmission cable modelling: Reactive power compensation and transient response studies," in IEEE 17th Workshop on Control and Modeling for Power Electronics, Jun. 2016, pp. 1-6.

[11] J. Song-Manguelle, M. H. Todorovic, S. Chi, S. K. Gunturi, and R. Datta, "Power transfer capability of HVAC cables for subsea transmission and distribution systems," IEEE Transactions on Industry Applications, vol. 50, no. 4, pp. 2382-2391, July 2014.

[12] J. Machado, M. V. Neves, and P. J. Santos, "Economic limitations of the HVAC transmission system when applied to offshore wind farms," in 9th International Conference on Compatibility and Power Electronics, Jun. 2015, pp. 69-75.

[13] K. Frey, K. Rudion, and J. Christian, "Automated operation approach for embedded HVDC links during (N-1)-conditions in the AC system," in IEEE International Energy Conference, Leuven, Belgium, Apr. 2016, pp. 1-6.

[14] K. Frey, P. Wiest, K. Rudion, and J. Christian, "Automated operation of parallel VSC HVDC links within an interconnected AC network," in IEEE Power and Energy Society General Meeting, Boston, MA, United states, Jul. 2016, pp. 1-5.

[15] L. Castaing, M.-S. Debry, G. Bareux, and O. Beck, "Optimal operation of HVDC links embedded in an AC network," in IEEE Grenoble Conference PowerTech, Grenoble, France, Jun. 2013, pp. 1-6.

[16] F. Capitanescu et al., "State-of-the-art, challenges, and future trends in security constrained optimal power flow," Electric Power Systems Research, vol. 81, no. 8, pp. 1731 - 1741, 2011.

[17] A. M. Geoffrion, "Generalized benders decomposition," Journal of Optimization Theory and Applications, vol. 10, no. 4, pp. 237-260, 1972

[18] A. Monticelli, M. V. F. Pereira, and S. Granville, "Security-constrained optimal power flow with post-contingency corrective rescheduling," IEEE Transactions on Power Systems, vol. 2, no. 1, pp. 175-180, Feb 1987.

[19] R. M. Tumilty, "Assessment of wind generation contribution to GB power system stability deliverable 5: Application notes for a representative dynamic model for the GB power system," University of Strathclyde, Tech. Rep., 2014.

[20] M. Belivanis and K. R. W. Bell, "Coordination of phase-shifting transformers to improve transmission network utilisation," in IEEE PES Innovative Smart Grid Technologies Conference Europe, Oct. 2010, pp. $1-6$.

[21] _ _ "Use of phase-shifting transformers on the transmission network in Great Britain," in 45th International Universities Power Engineering Conference, Aug. 2010, pp. 1-5.

[22] K. R. W. Bell and A. N. D. Tleis, "Test system requirements for modelling future power systems," in IEEE Power and Energy Society General Meeting, Jul. 2010, pp. 1-8.

[23] "Electricity Ten Year Statement: Appendix F Generation Data," National Grid Electricity Transmission, Tech. Rep., 2015.

[24] "Electricity Ten Year Statement: Appendix G Demand Data," National Grid Electricity Transmission, Tech. Rep., 2015.

[25] "Summer Outlook Report," National Grid Electricity Transmission, Tech. Rep., 2016.

[26] “Transmission Losses Report,” SP Energy Networks, Tech. Rep., 2015.

[27] National Grid Electricity Transmission, "Electricity Scenario Illustrator, Version 2," Internet, 2013.

[28] "Technical assessment of the operation of coal \& gas fired plants," Parsons Brinckerhoff, Tech. Rep., 2014.

[29] A. J. Mason, "OpenSolver: An open source add-in to solve linear and integer progammes in Excel," in Operations Research Proceedings 2011, ser. Operations Research Proceedings. Springer Berlin Heidelberg, 2012, pp. 401-406.

[30] W. E. Hart, J.-P. Watson, and D. L. Woodruff, "Pyomo: modeling and solving mathematical programs in Python," Mathematical Programming Computation, vol. 3, no. 3, pp. 219-260, 2011.

[31] J. Forrest and R. Lougee-Heimer, CBC User Guide. IBM Corporation, 2005.

[32] A. Wächter and L. T. Biegler, "On the implementation of an interiorpoint filter line-search algorithm for large-scale nonlinear programming," Mathematical Programming, vol. 106, no. 1, pp. 25-57, 2006. 